\title{
Obesity alters the in vivo mechanical response and biochemical properties of cartilage as measured by MRI
}

\author{
Amber T Collins ${ }^{1}$, Micaela L Kulvaranon ${ }^{1}$, Hattie C Cutcliffe ${ }^{1,2}$, Gangadhar M Utturkar', Wyatt A R Smith', \\ Charles E Spritzer ${ }^{4}$, Farshid Guilak ${ }^{5}$ and Louis E DeFrate 1,2,3* $^{*}$
}

\begin{abstract}
Background: Obesity is a primary risk factor for the development of knee osteoarthritis $(\mathrm{OA})$. However, there remains a lack of in vivo data on the influence of obesity on knee cartilage mechanics and composition. The purpose of this study was to determine the relationship between obesity and tibiofemoral cartilage properties.

Methods: Magnetic resonance images (3T) of cartilage geometry (double-echo steady-state) and T1rho relaxation of the knee were obtained in healthy subjects with a normal $(n=8)$ or high $(n=7)$ body mass index (BMI) before and immediately after treadmill walking. Subjects had no history of lower limb injury or surgery. Bone and cartilage surfaces were segmented and three-dimensional models were created to measure cartilage thickness and strain. T1rho relaxation times were measured before exercise in both the tibial and femoral cartilage in order to characterize biochemical composition. Body fat composition was also measured.

Results: Subjects with a high BMl exhibited significantly increased tibiofemoral cartilage strain and T1rho relaxation times $(P<0.05)$. Tibial pre-exercise cartilage thickness was also affected by BMI $(P<0.05)$. Correlational analyses revealed that pre-exercise tibial cartilage thickness decreased with increasing $B M I\left(R^{2}=0.43, P<0.01\right)$ and body fat percentage $\left(R^{2}=0.58, P<0.01\right)$. Tibial and femoral cartilage strain increased with increasing $B M I\left(R^{2}=0.45, P<0.01\right.$; $R^{2}=0.51, P<0.01$, respectively) and increasing body fat percentage $\left(R^{2}=0.40, P<0.05 ; R^{2}=0.38, P<0.05\right.$, respectively). Additionally, tibial T1rho was positively correlated with $B M I\left(R^{2}=0.39, P<0.05\right)$ and body fat percentage $\left(R^{2}=0.47, P<0.01\right)$.

Conclusions: Strains and T1rho relaxation times in the tibiofemoral cartilage were increased in high BMI subjects compared with normal BMI subjects. Additionally, pre-exercise tibial cartilage thickness decreased with obesity. Reduced proteoglycan content may be indicative of pre-symptomatic osteoarthritic degeneration, resulting in reduced cartilage thickness and increased deformation of cartilage in response to loading.
\end{abstract}

Keywords: Obesity, Cartilage, Magnetic resonance imaging (MRI), Proteoglycan, mechanobiology, stress test

\section{Background}

Obesity is a major risk factor for osteoarthritis (OA) [13 ] and the incidence of knee OA in obese individuals is four times greater than that in healthy weight controls [4]. Whereas the association between OA and obesity has been established, the mechanisms by which obesity

\footnotetext{
* Correspondence: lou.defrate@duke.edu

'Department of Orthopaedic Surgery, Duke University, Box 3093, Duke University Medical Center, Durham, NC 27710, USA

${ }^{2}$ Department of Biomedical Engineering, Duke University, Campus Box 90281, 101 Science Drive, Durham 27708, NC, USA

Full list of author information is available at the end of the article
}

increases the risk for OA are not well understood. Some studies attribute the increased risk of OA with obesity to increased joint loading due to elevated body mass [5, 6]; however, more recently, it has been suggested that a combination of biomechanical and metabolic factors, such as cartilage catabolism due to adipokine-related inflammation, plays an important role in this relationship $[7,8]$. Furthermore, the presence of $\mathrm{OA}$ in non-weight-bearing joints of obese subjects $[9,10]$ suggests that factors other than mechanical loading potentially contribute to disease progression. 
Nonetheless, there remains a lack of in vivo data describing the effects of obesity on cartilage composition and mechanical function. Although gait analysis studies can provide estimates of the loads experienced by the knee joint [11-13], it is unclear how these estimated loads relate to local in vivo cartilage deformation. Cartilage is a biphasic viscoelastic material due to the time-dependent exudation of water that occurs following mechanical loading of the tissue. Owing to the low permeability of the cartilage matrix, recovery of water back into the matrix once unloaded is not immediate [14-17]. Several studies have used magnetic resonance imaging (MRI) to characterize the in vivo deformation of cartilage by taking advantage of this time-dependent biomechanical recovery following loading [14-19]. Thus, this technique can be used to assess the effects of obesity on in vivo cartilage mechanics.

Previously, Widmyer et al. used MRI to show that increased body mass index (BMI) is associated with increased diurnal cartilage strains in the knee when compared with normal weighted controls; diurnal strain was defined as change in cartilage thickness from morning to evening [18]. However, it is unclear whether these increased cartilage strains associated with obesity are due solely to greater body mass or alterations in cartilage composition or both. Currently, there are limited in vivo data quantifying how obesity relates to alterations in cartilage composition and how these changes are related to altered mechanical function. Quantitative MRI techniques, such as T1rho-weighted imaging, have been used to quantify in vivo proteoglycan content in cartilage [20-24] and therefore can be used to assess changes in cartilage composition with obesity. The objective of this study was to assess how obesity alters both the in vivo mechanical function and composition of cartilage. We hypothesized that obesity is associated with a reduction in proteoglycan content, as evidenced by increased T1rho relaxation times. Additionally, we hypothesized that these alterations in composition result in decreased cartilage stiffness, which will be reflected by increased in vivo cartilage strain in response to mechanical loading. Our overall hypothesis is that obese subjects exhibit "pre-OA" changes in both articular cartilage composition and mechanical function that precede the onset of symptomatic OA.

\section{Methods}

\section{Subject recruitment}

Following approval by the institutional review board of Duke University Medical Center, eight subjects (five males and three females; mean age 30 years, range 2343; mean height 70 in., range 64-74) with a normal BMI (mean 22.2; range 18-25) and seven subjects (three males and four females; mean age 32, range 22-45; mean height 66 in., range 63-71) with a high BMI (mean 32.8; range 30-36), who were otherwise healthy, were recruited for participation in this study. The normal BMI and high BMI groups were statistically significantly different with regard to BMI $(P<0.0001, t$ test $)$. However, no statistically significant differences were detected with regard to age ( $P=0.64, t$ test), height $(P=0.08, t$ test), or the distribution of males and females between groups $(P$ $=0.613$, Fisher's exact test). Previous work from our lab investigating cartilage strains in healthy subjects tested eight subjects and found significant changes in cartilage thickness as a result of treadmill walking [15]. Therefore, we aimed to recruit and test a similar number of subjects per group in the present study. All subjects provided informed written consent before beginning the study. Subjects were excluded if they had a history of lower limb injury, surgery, or symptoms related to OA.

\section{Study procedure}

In order to minimize the effect of diurnal cartilage loading $[14,18,19]$, subjects were tested early in the morning and instructed not to perform any strenuous activities on the day prior to and the morning of testing. Upon arrival, subjects lay supine for $45 \mathrm{~min}$ prior to the pre-exercise MRI scan to allow their knee cartilage to relax to its baseline, unloaded state in a room adjacent to the MRI scanner [19]. Following this relaxation period, subjects were transported to the MRI scanner in a wheelchair. Pre-exercise MRI images of each subject's right knee were taken in the sagittal plane using a $3.0 \mathrm{~T}$ MRI scanner with an eight-channel knee coil [25] (Trio Tim, Siemens Medical Solutions USA, Malvern, PA, USA). A three-dimensional (3D) double-echo steady-state (DESS) sequence-flip angle: $25^{\circ}$; echo time (TE): $6 \mathrm{~ms}$; repetition time (TR): $17 \mathrm{~ms}$; field of view (FOV): $16 \times 16 \mathrm{~cm}$; matrix: $512 \times 512$ pixels; resolution: $0.3 \times 0.3 \times 1.0 \mathrm{~mm}$-was used to obtain anatomical images of the bones and articular cartilage, allowing for pre-exercise cartilage thickness measurements. A T1rho-weighted imaging sequence with a 3D fast imaging with steady-state precession (FISP) acquisitionflip angle: $15^{\circ}$; TE: $5.9 \mathrm{~ms}$; TR: $3500 \mathrm{~ms}$; FOV: $14 \times 14$ $\mathrm{cm}$; matrix: $256 \times 256$ pixels; resolution: $1.1 \times 0.5 \times$ $3.0 \mathrm{~mm}$; B1: $500 \mathrm{~Hz}$; spin lock time (TSL): 5, 10, 40, $80 \mathrm{~ms}$-was collected in order to assess proteoglycan content within the cartilage $[14,15]$. Following the pre-exercise MRI scan, subjects were transported by wheelchair to an adjacent room where they walked on a treadmill for $20 \mathrm{~min}$. Walking speed was normalized to the subject's leg length using the Froude number $(\mathrm{Fr})$ $\left(\mathrm{Fr}=\mathrm{v}^{2} /(\mathrm{L} \times \mathrm{g})\right)[26]$, which uses leg length (L) as measured from the greater trochanter of the femur to the ground surface, and the gravitational constant $(\mathrm{g}=$ $9.8 \mathrm{~m} / \mathrm{s}^{2}$ ) in order to calculate a normalized walking 
speed (v). Subjects walked at a Froude number of 0.25 , which corresponds to an adult walking at a comfortable pace [26]. Subjects also wore a pedometer to record the number of steps taken during their 20-min walk. Immediately following exercise, subjects were transported back to the MRI scanner for a post-exercise DESS sequence scan which was used to measure post-exercise cartilage thickness. Lastly, each subject's body composition (weight and body fat percentage) was measured immediately following the post-exercise MRI scan by using a bioelectrical impedance scale (InBody230, BioSpace Inc., Cerritos, CA, USA) [27].

\section{Data analysis}

The tibial and femoral bony and articular cartilage surfaces were segmented on the DESS images by using solid modeling software (Rhinoceros; Robert McNeel \& Associates, Seattle, WA, USA) [14-16]. Segmentations from each DESS MRI slice were compiled to create 3D mesh models of the proximal tibia and distal femur as well as of both the associated articulating surfaces. The pre-exercise and post-exercise models were registered together by using an iterative closest-point algorithm, allowing for site-specific comparisons of cartilage thickness between the pre- and post-exercise scans (Geomagic Studio; Geomagic, 3D Systems, Valencia, CA, USA) [28]. Cartilage thickness maps were generated by calculating the distance from each vertex on the cartilage surface mesh to its nearest vertex on the corresponding bone surface mesh (Fig. 1a). Thickness measurements of both the tibial and femoral cartilage were averaged within uniformly spaced points, each with a radius of $2.5 \mathrm{~mm}$. Eighteen points were placed on the tibial cartilage ( 9 points on each tibial plateau), and 36 points were placed on the femoral cartilage (18 points on each condyle) (Fig. 1b) [29]. Strain at each of these points was calculated as the difference between the pre- and post-exercise thickness, divided by the pre-exercise thickness [14]. These strains were averaged to generate mean cartilage strains representing strain across the tibial and femoral cartilage. The methodology used in the present study has been previously validated to measure cartilage thickness in the tibiofemoral joint to within a resolution of $1 \%[19,29]$.

T1rho relaxation time maps were generated from the pre-exercise T1rho MRI images. The tibial and femoral cartilage were manually segmented from the TSL $=5 \mathrm{~ms}$ image (Fig. 2). For each voxel within the segmented cartilage regions, T1rho relaxation times were calculated by assessing the exponential decay of the MRI signal intensity with increasing spin lock time (TSL). This was done by fitting the following equation [30]:

$$
S(T S L)=S_{0} e^{-T S L / T_{1 \rho}}
$$

Here, S(TSL) represents signal intensity for a given voxel, $S_{0}$ represents initial signal intensity, and TSL represents spin lock time. T1rho relaxation times of all voxels within the segmented region of the tibial cartilage were averaged, as were the relaxation times of all voxels of the femoral cartilage.

\section{T1rho repeatability analysis}

The repeatability of this technique was assessed by comparing baseline tibial and femoral cartilage T1rho relaxation times acquired from four male test subjects (mean age 32, range 27-40; mean BMI 22.3, range 18-24) during two separate MRI sessions which occurred within a 2 -week period. Subjects were tested at 8 am with a 45 -min rest period prior to each testing session. The coefficient of variation of the acquired T1rho relaxation times was determined to be $1.4 \%$.
A

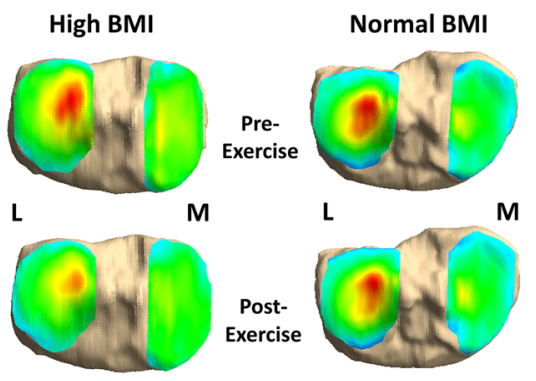

B

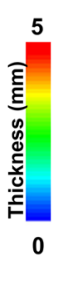

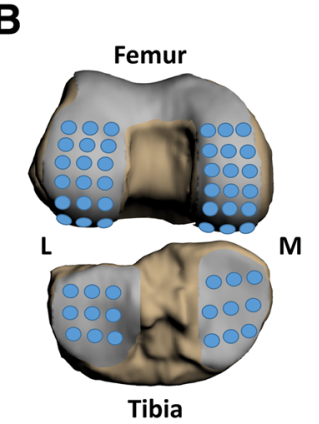

Fig. 1 a Representative tibial cartilage thickness maps from a high body mass index (BMI) subject and a normal BMI subject. The color thickness maps demonstrate greater changes in the high BMI subject compared with the normal BMI subject following the 20-min walking task in both the medial $(M)$ and lateral $(L)$ aspects of the tibial cartilage. $\mathbf{b}$ Femur and tibia with articular cartilage surfaces demonstrating the grid point sampling locations. The tibial cartilage surfaces were sampled from 18 points, and the femoral cartilage surfaces were sampled from 36 points 


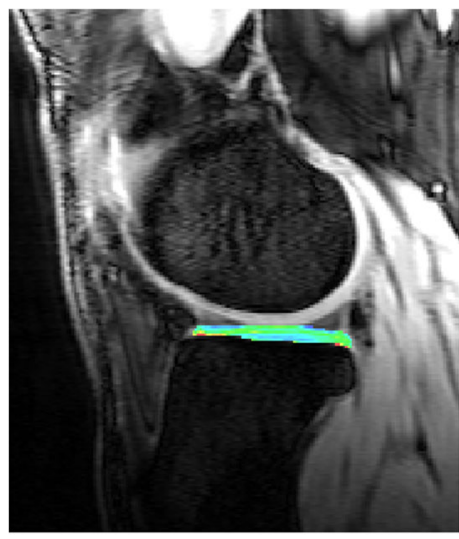

High BMI Subject

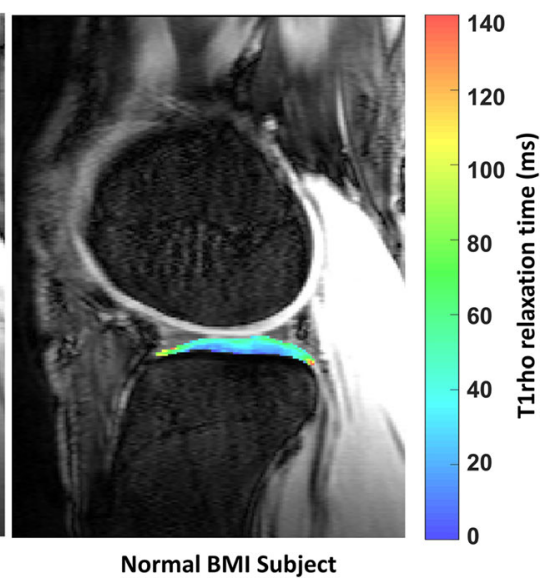

Fig. 2 Color map of tibial cartilage T1rho relaxation times in one representative high body mass index (BMI) subject and one representative normal BMI subject

\section{Statistical analysis}

Two-way repeated measures analysis of variance (ANOVA) was performed to determine the influence of BMI (high versus normal) and location (femur versus tibia) on cartilage strain, T1rho relaxation time, and pre-exercise cartilage thickness. Fisher's least significant difference (LSD) test was used for post hoc comparisons in cases where the ANOVA indicated a significant interaction between variables. Additionally, simple linear regressions were performed to analyze relationships between body composition (BMI and body fat percentage) and tibial and femoral cartilage properties (cartilage thickness, strain, and T1rho relaxation time). Statistical significance was defined as a $P$ value of less than 0.05 .

\section{Results}

Overall, high BMI subjects had significantly decreased resting tibial cartilage thickness $(P<0.05$, Fig. 3a) compared with normal BMI subjects. Additionally, high BMI subjects had increased compressive tibiofemoral cartilage strains compared with normal BMI subjects ( $P$ $<0.01$, Fig. 3b). Finally, high BMI subjects had elevated tibiofemoral T1rho relaxation times compared with normal BMI subjects $(P=0.03$, Fig. $3 c)$, and femoral T1rho relaxation times were greater than tibial T1rho relaxation times $(P<0.01)$.

As expected, BMI was significantly correlated with body fat percentage $\left(P<0.05, \mathrm{R}^{2}=0.79\right)$. Additionally, tibial pre-exercise cartilage thickness was negatively correlated with BMI $\left(P<0.01, \mathrm{R}^{2}=0.43\right.$, Fig. $\left.4 \mathrm{a}\right)$, with $12 \%$ thinner cartilage in high BMI subjects compared with normal BMI subjects. Pre-exercise tibial cartilage thickness was also negatively correlated with body fat percentage $\left(P<0.01, \mathrm{R}^{2}=0.58\right.$, Fig. 4b). Tibial cartilage strain was positively correlated with BMI $\left(P<0.01, \mathrm{R}^{2}=\right.$ 0.45 , Fig. 5a), with a nearly fourfold increase in high BMI subjects compared with normal BMI subjects. Tibial strain was also positively correlated with body fat percentage $\left(P<0.05, \mathrm{R}^{2}=0.40\right.$, Fig. $\left.5 \mathrm{~b}\right)$.

Femoral pre-exercise cartilage thickness was not significantly correlated to either BMI $\left(P=0.79, \mathrm{R}^{2}=0.006\right)$ or body fat percentage $\left(P=0.71, \mathrm{R}^{2}=0.011\right)$. However, femoral cartilage strain was positively correlated with BMI $\left(P<0.01, \mathrm{R}^{2}=0.51\right.$, Fig. $\left.5 \mathrm{c}\right)$, again with a nearly

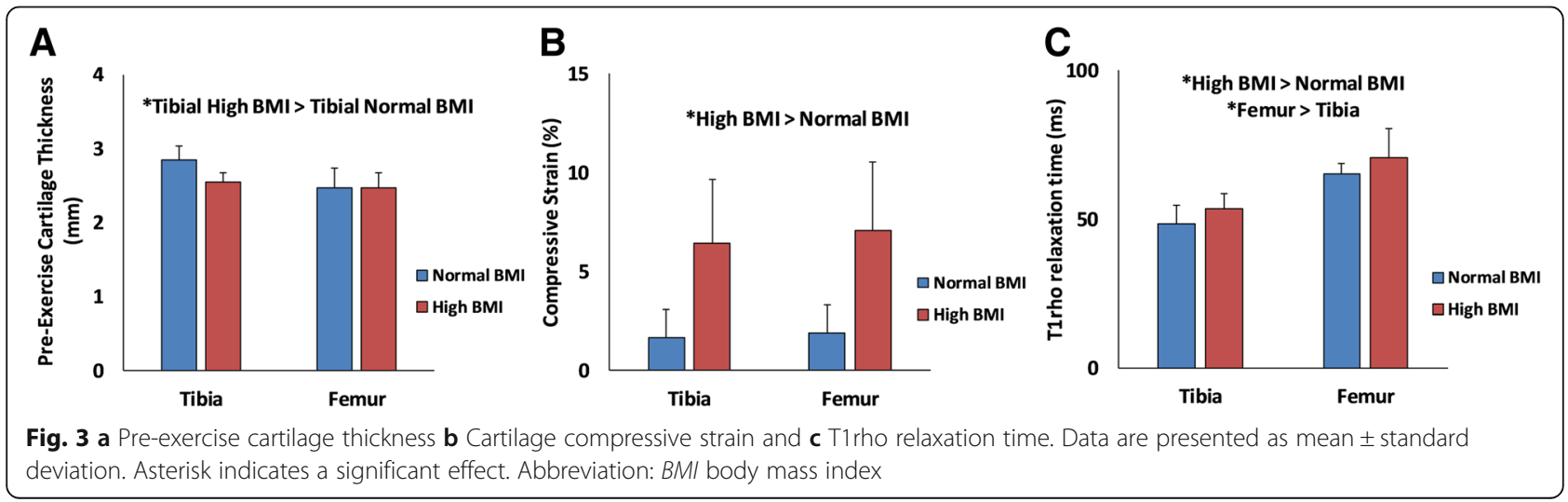


fourfold increase in high BMI subjects compared with normal BMI subjects. Additionally, femoral strain was also positively correlated with body fat percentage ( $P$ $<0.05, R^{2}=0.38$, Fig. 5d).

Pre-exercise tibial T1rho relaxation times were significantly correlated with BMI $\left(P<0.05, R^{2}=0.39\right.$, Fig. $\left.6 a\right)$, and high BMI subjects had 13\% greater T1rho relaxation times compared with normal BMI subjects. Likewise, pre-exercise tibial T1rho was significantly correlated with body fat percentage $\left(P<0.01, \mathrm{R}^{2}=0.47\right.$, Fig. $\left.6 \mathrm{~b}\right)$. However, pre-exercise femoral T1rho relaxation times were not correlated with BMI $\left(P=0.31, \mathrm{R}^{2}=0.08\right)$ or body fat percentage $\left(P=0.6, \mathrm{R}^{2}=0.08\right.$, Fig. 6 c, d $)$.

\section{Discussion}

Obesity is a major risk factor for the development of knee OA; however, the relative contribution of biochemical and biomechanical factors to the obesity-OA relationship is unclear. In order to better understand the mechanisms by which obesity leads to OA, this study aimed to quantify both biomechanical and biochemical changes in tibiofemoral articular cartilage that occur with high BMI and increased body fat percentage. Specifically, this study explored the influence of obesity on changes in both in vivo cartilage strain in response to an acute, dynamic loading task (20-min treadmill walk) as well as baseline cartilage proteoglycan content using T1rho relaxation imaging. Our study found that obese subjects, who had no history of joint injury or surgery and no symptoms of knee OA, exhibited lower pre-exercise tibial cartilage thickness as well as greater tibiofemoral cartilage compressive strain following loading. Additionally, we found that high BMI subjects exhibited increased tibiofemoral T1rho relaxation times, which may be indicative of decreased proteoglycan content within the cartilage [22, 31, 32].

Increased tibial and femoral in vivo cartilage strains demonstrated with increased BMI are consistent with previous work from our lab which investigated diurnal changes in cartilage thickness [18]. Widmyer et al. found that over the course of normal daily activities, subjects with a high BMI (25-31) had significantly higher compressive strains in the tibial cartilage compared with those with a normal BMI (18.5-24.9) [18]. Whereas Widmyer et al. compared cartilage thickness in the morning and evening to obtain measures of diurnal strain, the current study demonstrated similar changes in tibial and femoral cartilage thickness between BMI groups as a result of a controlled and shorter duration loading activity. Specifically, this study investigated cartilage strains in response to treadmill walking for $20 \mathrm{~min}$ at a speed normalized to each subject's lower limb length by using the Froude number (Fr) [26]. Controlling for walking speed across subjects by using the Froude number was important in this study as previous work has shown that given the option of self-selected walking speed, obese individuals walk slower than lean individuals, thus possibly influencing ground reaction forces and subsequent loads experienced by the knee joint [33].

Gait analysis studies have been previously used to approximate joint reaction forces in response to obesity [34-36]. For example, Harding et al. demonstrated that
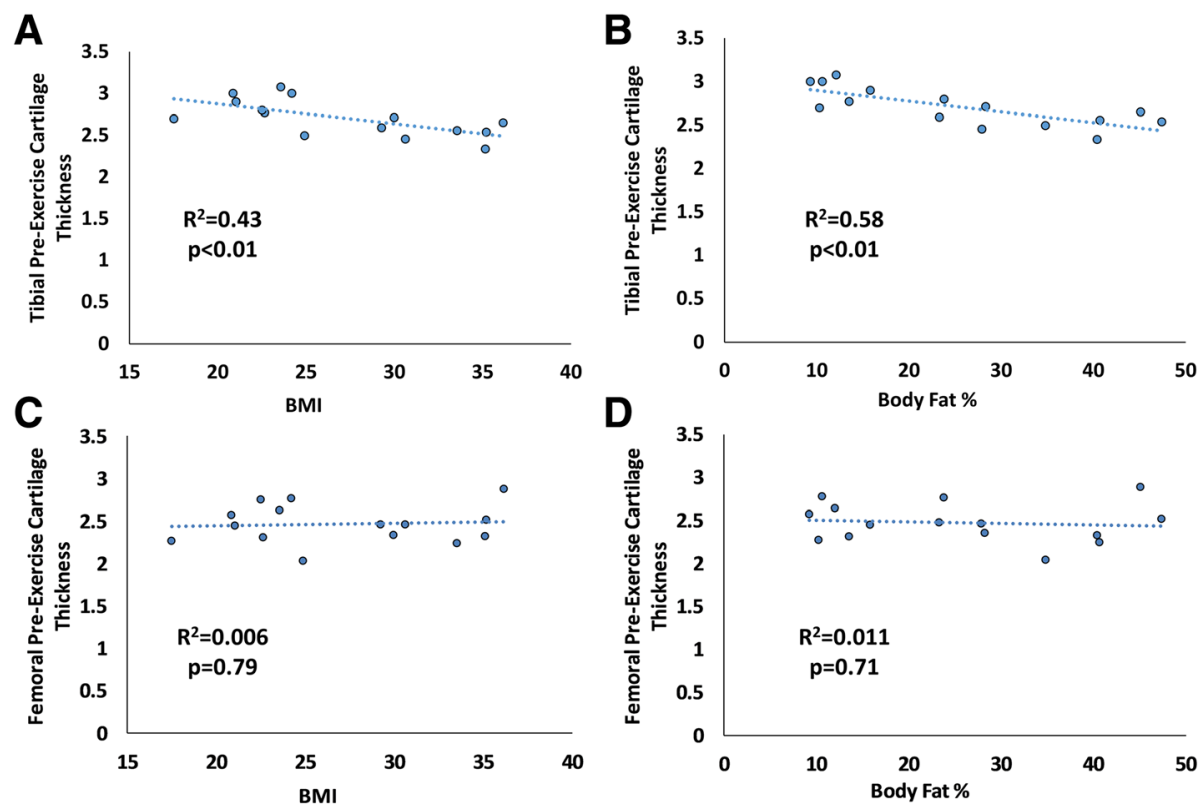

Fig. 4 a, b Tibial cartilage pre-exercise thickness was significantly correlated with body mass index (BMI) $(P<0.01)$ and body fat percentage $(P$ $<0.01)$. , d Femoral cartilage pre-exercise thickness was not significantly correlated with BMI $(P=0.79)$ or body fat percentage $(P=0.71)$ 

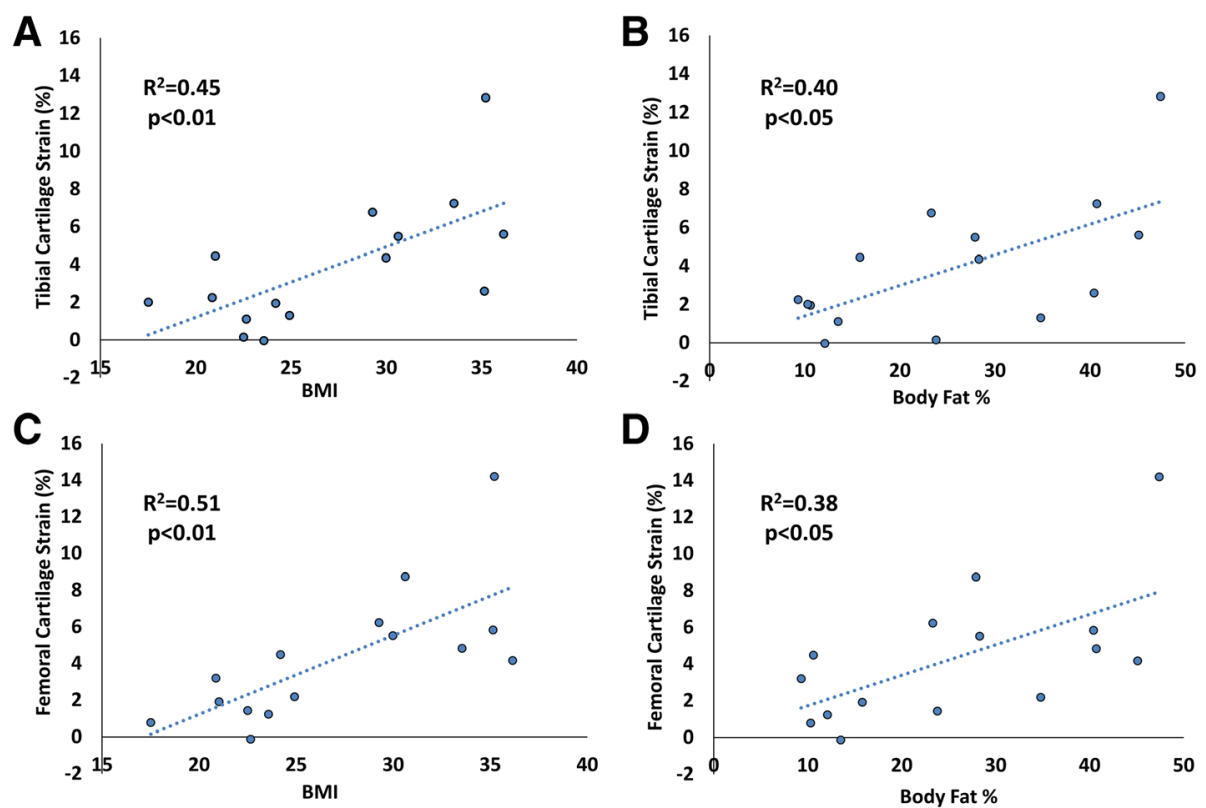

Fig. 5 a, b Tibial cartilage compressive strain was significantly correlated with body mass index (BMI) $(P<0.01)$ and body fat percentage $(P<0.05)$. c, d Femoral cartilage strain was also significantly correlated with BMI $(P<0.01)$ and body fat percentage $(P<0.05)$

high BMI is associated with higher absolute tibiofemoral compressive forces by using a sagittal plane contact force model [34]. In a separate weight loss study, DeVita et al. demonstrated that weight loss of approximately $34 \%$ of initial body weight reduced maximum compressive forces in the knee as measured through gait analysis techniques, but these changes were later attenuated by gait adaptations following weight loss [35]. Additionally, Liukkonen et al. showed that weight loss induced by bariatric surgery altered knee kinetics and kinematics during gait [36]. In general, such gait analysis studies provide a wealth of information regarding knee joint
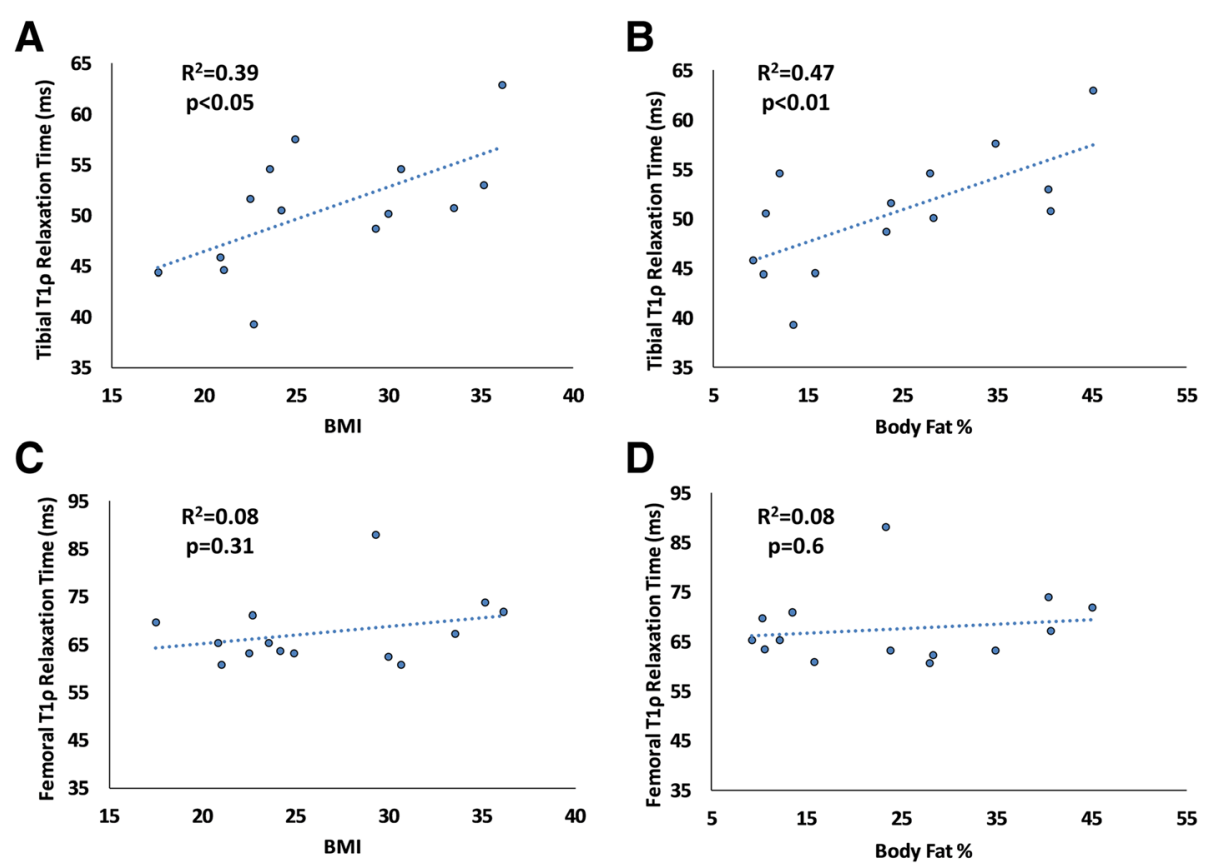

Fig. $\mathbf{6}$ a, b Tibial T1rho relaxation time was significantly correlated with body mass index (BMI) $(P<0.05)$ and body fat percentage $(P<0.01)$. $\mathbf{c}, \mathbf{d}$ Femoral T1rho relaxation time was not significantly correlated with BMI $(P=0.31)$ or body fat percentage $(P=0.6)$ 
loading in various populations. The present study further suggests that, in addition to potentially altering joint loading, obesity may be associated with presymptomatic alterations in the mechanical response and composition of cartilage.

In addition to changes in the mechanical function of the cartilage, we observed alterations in cartilage biochemical composition occurring with obesity. Specifically, we observed decreased proteoglycan content in the tibiofemoral cartilage of high BMI subjects as measured using T1rho imaging. Consistent with these findings, one previous study used delayed gadolinium-enhanced magnetic resonance imaging of cartilage (dGEMRIC) to estimate proteoglycan content in the articular cartilage of subjects before and after a weight loss program [37]. They concluded that weight loss was associated with protective effects on proteoglycan content and cartilage thickness [37]. Changes in cartilage composition have important implications for the mechanical behavior of cartilage and thus the development of OA [22, 31]. Specifically, decreased proteoglycan content has been shown to be related to decreased aggregate modulus, or stiffness, of cartilage resulting in increased deformation in response to load [31, 38]. Structural modifications to cartilage components such as proteoglycan loss may directly affect the ability of cartilage to withstand and transfer load. To this point, one study found that, in a diet-induced mouse model, high fat gain was associated with cartilage proteoglycan loss and reduced the aggregate modulus of cartilage in high fat-fed mice [39]. These results demonstrate that a high-fat diet may alter the material properties of cartilage by reducing proteoglycan content and decreasing tissue stiffness, potentially leading to increased deformation due to mechanical loading as observed in the present study. Importantly, chondrocyte metabolism is closely related to its mechanical environment [40], and several studies have shown that hyperphysiologic magnitudes of cartilage loading can lead to decreased synthesis of extracellular matrix components, increased production of pro-inflammatory cytokines, and potentially cell death [41-44]. Thus, altered mechanical properties related to changes in biochemical composition can change the mechanical environment experienced by chondrocytes, thus changing their metabolic activity and potentially contributing to a progressive cycle of degeneration [45]. Further support for this hypothesis is provided by evidence of decreased pre-exercise tibial cartilage thickness observed in obese subjects in this study, which may be indicative of cartilage degeneration.

The exact mechanism of obesity-related OA is unclear but may be the result of alterations in both the local mechanical loading and inflammatory environments. Specifically, it is possible that local mechanical factors, such as increased joint loading, exacerbate the effects of inflammatory cytokines (either systemic or localized), thus furthering the cycle of cartilage degeneration. Additionally, obesity is considered a systemic inflammatory disease which has been shown to induce OA in non-weight-bearing joints such as the wrist and hand $[10,46]$. Recent studies suggest that metabolic factors occurring with obesity alter the activity of inflammatory cytokines that are associated with OA $[47,48]$. Specifically, increased pro-inflammatory biomarkers-tumor necrosis factor alpha (TNF- $\alpha$ ), interleukin-1 beta (IL-1 $\beta$ ), and IL-6-have been observed in obese children with no comorbidities [47] as well as in obese patients with OA [49]. However, diet and exercise can reduce these cytokine levels $[49,50]$. Such increased inflammatory cytokine activity can induce chondrocyte catabolism, resulting in degenerative changes such as decreased proteoglycan content $[51,52]$. Similarly, the increased tibial T1rho relaxation time (corresponding to decreased proteoglycan content) with both increasing BMI and body fat percentage observed in the present study may be due to increased activity of inflammatory cytokines [51, 52]. The stronger correlation of tibial T1rho relaxation time with body fat percentage than with BMI demonstrated in this study may be suggestive of adipose-related inflammation playing a greater role than mechanical loading in the obesity-cartilage degeneration relationship. Future studies may further investigate the relative contributions of body mass and body fat percentage on cartilage deformation and composition.

In this study, measures of cartilage strain may have been underestimated because of inadequate recovery of the cartilage prior to the pre-exercise MRI scan or because of partial recovery prior to the post-exercise MRI scan. Although subjects were asked to refrain from strenuous activity $24 \mathrm{~h}$ prior to and the morning of testing, and a 45-min period of supine resting time was included to allow cartilage thickness to reach its baseline unloaded state, it is possible that the cartilage was not fully recovered prior to pre-exercise imaging. Additionally, the period between completion of the walking activity and the post-exercise MRI scan was less than $4 \mathrm{~min}$. Previous work has demonstrated that cartilage volume recovers to about $50 \%$ of its original volume after $45 \mathrm{~min}$ of being unloaded [53]. Therefore, it is possible that some cartilage recovery occurred during this time; however, there was no difference between groups either in the study tasks completed or in the time between the completion of the walking activity and the post-exercise MRI scan. Nonetheless, cartilage recovery occurring within this 4-min period may result in underestimations of cartilage strain.

\section{Conclusions}

The present study demonstrates a significant relationship between body composition and the biomechanical 
and biochemical properties of cartilage. We found that a short treadmill walking task resulted in greater tibiofemoral cartilage strains in subjects with a high BMI as compared with subjects with a normal BMI. Additionally, we found that tibiofemoral T1rho relaxation times increased in high BMI subjects, indicative of a decrease in cartilage proteoglycan concentration. Taken together with our previous work [18], these data indicate that the changes in cartilage strain observed here may be the result of alterations in both biomechanics (that is, increased joint load) and cartilage composition (loss of proteoglycan) leading to changes in mechanical properties. Importantly, decreases in proteoglycan content and cartilage thickness in obese subjects may be indicative of a "pre-osteoarthritic" state of cartilage. Characterizing the effects of BMI and body fat percentage on in vivo cartilage properties is a critical first step in understanding the mechanisms by which obesity alters the mechanical and biochemical properties of cartilage, thus contributing to the initiation and progression of knee OA.

\section{Abbreviations}

3D: Three-dimensional; ANOVA : Analysis of variance; BMI: Body mass index; DESS: Double-echo steady-state; FOV: Field of view; Fr: Froude number; IL: Interleukin; MRI: Magnetic resonance imaging; OA: Osteoarthritis; TE: Echo time; TNF: Tumor necrosis factor alpha; TR: Repetition time; TSL: Spin lock time

\section{Acknowledgments}

The authors would like to thank Jean Shaffer at the Duke University Center for Advanced Magnetic Resonance Development (Durham, NC, USA) for technical assistance.

\section{Funding}

This study was supported by the National Institutes of Health, specifically the National Institute of Arthritis and Musculoskeletal and Skin Diseases and the National Institute on Aging (AR066477, AR065527, AR50245, and AG46927).

\section{Availability of data and materials}

Owing to privacy of subject information, the datasets generated and analyzed during this study are not publicly available but are available from the corresponding author on reasonable request.

\section{Authors' contributions}

AC contributed to this article through study design, collection of all data, analysis and interpretation of data, and the writing of the article. MK, $\mathrm{HC}$, and WS contributed to this article through data analysis and editing of the article. GU and CS contributed to this article through analysis and interpretation of data and editing of the article. FG and LD contributed to this article through the conception and design of the study, analysis and interpretation of data, and the writing of the article. All authors contributed to this work, agree with its contents, and have provided their final approval for submission.

\section{Ethics approval and consent to participate}

This study was approved by the Duke University Medical Center Institutional Review Board, and all subjects gave their informed consent to participate in the study.

\section{Consent for publication}

Not applicable.

\section{Competing interests}

The authors declare that they have no competing interests.

\section{Publisher's Note}

Springer Nature remains neutral with regard to jurisdictional claims in published maps and institutional affiliations.

\section{Author details}

'Department of Orthopaedic Surgery, Duke University, Box 3093, Duke University Medical Center, Durham, NC 27710, USA. 'Department of Biomedical Engineering, Duke University, Campus Box 90281, 101 Science Drive, Durham 27708, NC, USA. ${ }^{3}$ Department of Mechanical Engineering and Materials Science, Duke University, Campus Box 90300, Hudson Hall, Durham 27708, NC, USA. ${ }^{4}$ Department of Radiology, Duke University, Box 3808, Duke University Medical Center, Durham 27710, NC, USA. ${ }^{5}$ Department of Orthopaedic Surgery, Washington University and Shriners Hospitals for Children, Campus Box 8233, Couch Research Building, Room 3121, St. Louis 63110, MO, USA.

Received: 25 May 2018 Accepted: 19 September 2018

Published online: 17 October 2018

\section{References}

1. Felson DT. Relation of obesity and of vocational and avocational risk factors to osteoarthritis. J Rheumatol. 2005:32:1133-5. http://www.ncbi.nlm.nih.gov/ pubmed/15977343.

2. Runhaar J, Koes BW, Clockaerts S, Bierma-Zeinstra SM. A systematic review on changed biomechanics of lower extremities in obese individuals: a possible role in development of osteoarthritis. Obes Rev. 2011;12:1071-82. https://doi.org/10.1111/j.1467-789X.2011.00916.X.

3. Felson DT. Weight and osteoarthritis. J Rheumatol Suppl. 1995;43:7-9. http://www.ncbi.nlm.nih.gov/pubmed/7752143.

4. Murphy L, Schwartz TA, Helmick CG, Renner JB, Tudor G, Koch G, et al. Lifetime risk of symptomatic knee osteoarthritis. Arthritis Rheum. 2008;59: 1207-13. https://doi.org/10.1002/art.24021.

5. Powell A, Teichtahl AJ, Wluka AE, Cicuttini FM. Obesity: a preventable risk factor for large joint osteoarthritis which may act through biomechanical factors. Br J Sports Med. 2005;39:4-5. https://doi.org/10.1136/bjsm.2004.011841.

6. Felson DT. Does excess weight cause osteoarthritis and, if so, why? Ann Rheum Dis. 1996;55:668-70. http://www.ncbi.nlm.nih.gov/pubmed/8882146.

7. Griffin TM, Guilak F. Why is obesity associated with osteoarthritis? Insights from mouse models of obesity. Biorheology. 2008:45:387-98. http://www. ncbi.nlm.nih.gov/pubmed/18836239.

8. Aspden RM. Obesity punches above its weight in osteoarthritis. Nat Rev Rheumatol. 2011;7:65-8. https://doi.org/10.1038/nrrheum.2010.123.

9. Grotle M, Hagen KB, Natvig B, Dahl FA, Kvien TK. Obesity and osteoarthritis in knee, hip and/or hand: an epidemiological study in the general population with 10 years follow-up. BMC Musculoskelet Disord. 2008;9:132. https://doi.org/10.1186/1471-2474-9-132.

10. Oliveria SA, Felson DT, Cirillo PA, Reed Jl, Walker AM. Body weight, body mass index, and incident symptomatic osteoarthritis of the hand, hip, and knee. Epidemiology. 1999;10:161-6. https:/www.ncbi.nlm.nih.gov/pubmed/10069252.

11. Hurwitz D, Sumner D, Andriacchi T, Sugar D. Dynamic knee loads during gait predict proximal tibial bone distribution. J Biomech. 1998;31:423-30.

12. Landry SC, McKean KA, Hubley-Kozey CL, Stanish WD, Deluzio KJ. Knee biomechanics of moderate OA patients measured during gait at a selfselected and fast walking speed. J Biomech. 2007:40:1754-61. http://www. ncbi.nlm.nih.gov/entrez/query.fcgi?cmd=Retrieve\&db=PubMed\&dopt= Citation\&list_uids $=17084845$.

13. Fregly BJ, D'Lima DD, Colwell CW Jr. Effective gait patterns for offloading the medial compartment of the knee. J Orthop Res. 2009;27:1016-21. https://doi.org/10.1002/jor.20843.

14. Cher WL, Utturkar GM, Spritzer CE, Nunley JA, DeFrate LE, Collins AT. An analysis of changes in in vivo cartilage thickness of the healthy ankle following dynamic activity. J Biomech. 2016;49:3026-30. https://doi.org/10. 1016/j.jbiomech.2016.05.030.

15. Lad NK, Liu B, Ganapathy PK, Utturkar GM, Sutter EG, Moorman CT 3rd, et al. Effect of normal gait on in vivo tibiofemoral cartilage strains. J Biomech. 2016;49:2870-6. https://doi.org/10.1016/j.jbiomech.2016.06.025.

16. Liu B, Lad NK, Collins AT, Ganapathy PK, Utturkar GM, McNulty AL, et al. In vivo tibial cartilage strains in regions of cartilage-to-cartilage contact and cartilage-to-meniscus contact in response to walking. Am J Sports Med. 2017:45:2817-23. https://doi.org/10.1177/0363546517712506. 
17. Eckstein F, Lemberger B, Gratzke C, Hudelmaier M, Glaser C, Englmeier KH, et al. In vivo cartilage deformation after different types of activity and its dependence on physical training status. Ann Rheum Dis. 2005;64:291-5. https://doi.org/10.1136/ard.2004.022400.

18. Widmyer MR, Utturkar GM, Leddy HA, Coleman JL, Spritzer CE, Moorman CT $3 r d$, et al. High body mass index is associated with increased diurnal strains in the articular cartilage of the knee. Arthritis Rheum. 2013;65:2615-22. https://doi.org/10.1002/art.38062.

19. Coleman JL, Widmyer MR, Leddy HA, Utturkar GM, Spritzer CE, Moorman CT 3rd, et al. Diurnal variations in articular cartilage thickness and strain in the human knee. J Biomech. 2013;46:541-7. https://doi.org/10.1016/j.jbiomech. 2012.09.013

20. Tsushima H, Okazaki K, Takayama Y, Hatakenaka M, Honda H, Izawa T, et al Evaluation of cartilage degradation in arthritis using T1rho magnetic resonance imaging mapping. Rheumatol Int. 2012;32:2867-75. https://doi. org/10.1007/s00296-011-2140-3

21. Li X, Benjamin Ma C, Link TM, Castillo DD, Blumenkrantz G, Lozano J, et al. In vivo T(1rho) and T(2) mapping of articular cartilage in osteoarthritis of the knee using 3 T MRI. Osteoarthritis Cartilage. 2007;15:789-97. https://doi.org/ 10.1016/j.joca.2007.01.011.

22. Keenan KE, Besier TF, Pauly JM, Han E, Rosenberg J, Smith RL, et al. Prediction of glycosaminoglycan content in human cartilage by age, T1rho and T2 MRI. Osteoarthritis Cartilage. 2011;19:171-9. https://doi.org/10.1016/j. joca.2010.11.009.

23. Li X, Pai A, Blumenkrantz G, Carballido-Gamio J, Link T, Ma B, et al. Spatial distribution and relationship of T1rho and $T 2$ relaxation times in knee cartilage with osteoarthritis. Magn Reson Med. 2009;61:1310-8. https://doi. org/10.1002/mrm.21877.

24. Souza RB, Kumar D, Calixto N, Singh J, Schooler J, Subburaj K, et al. Response of knee cartilage T1rho and T2 relaxation times to in vivo mechanical loading in individuals with and without knee osteoarthritis. Osteoarthritis Cartilage. 2014;22:1367-76. https://doi.org/10.1016/j.joca.2014.04.017.

25. Taylor KA, Cutcliffe HC, Queen RM, Utturkar GM, Spritzer CE, Garrett WE, et al. In vivo measurement of $A C L$ length and relative strain during walking. J Biomech. 2013;46:478-83. https://doi.org/10.1016/j.jbiomech.2012.10.031.

26. Alexander RM, Jayes AS. A dynamic similarity hypothesis for the gaits of quadrupedal mammals. J Zool. 1983;201:135-52 <Go to ISI>://WOS: A1983RH79100010.

27. von Hurst PR, Walsh DCl, Conlon CA, Ingram M, Kruger R, Stonehouse W. Validity and reliability of bioelectrical impedance analysis to estimate body fat percentage against air displacement plethysmography and dual-energy X-ray absorptiometry. Nutr Diet. 2016;73:197-204. https://doi.org/10.1111/ 1747-0080.12172.

28. Okafor EC, Utturkar GM, Widmyer MR, Abebe ES, Collins AT, Taylor DC, et al. The effects of femoral graft placement on cartilage thickness after anterior cruciate ligament reconstruction. J Biomech. 2014;47:96-101. https://doi.org/ 10.1016/j.jbiomech.2013.10.003.

29. Van de Velde SK, Bingham JT, Hosseini A, Kozanek M, DeFrate LE, Gill TJ, et al. Increased tibiofemoral cartilage contact deformation in patients with anterior cruciate ligament deficiency. Arthritis Rheum. 2009;60:3693-702. https://doi.org/10.1002/art.24965.

30. Borthakur A, Wheaton A, Charagundla SR, Shapiro EM, Regatte RR, Akella SV, et al. Three-dimensional T1rho-weighted MRI at 1.5 Tesla. Journal of magnetic resonance imaging. JMRI. 2003;17:730-6. https://doi.org/10.1002/ jmri.10296.

31. Hatcher CC, Collins AT, Kim SY, Michel LC, Mostertz WC 3rd, Ziemian SN, et al Relationship between T1rho magnetic resonance imaging, synovial fluid biomarkers, and the biochemical and biomechanical properties of cartilage. J Biomech. 2017;55:18-26. https://doi.org/10.1016/j.jbiomech.2017.02.001.

32. Wheaton AJ, Dodge GR, Borthakur A, Kneeland JB, Schumacher HR, Reddy R. Detection of changes in articular cartilage proteoglycan by $\mathrm{T}(1 \mathrm{rho})$ magnetic resonance imaging. J Orthop Res. 2004;23:102-8. https://doi.org/ 10.1016/j.orthres.2004.06.015

33. DeVita P, Hortobagyi T. Obesity is not associated with increased knee joint torque and power during level walking. J Biomech. 2003;36:1355-62. https://www.ncbi.nlm.nih.gov/pubmed/12893044.

34. Harding GT, Dunbar MJ, Hubley-Kozey CL, Stanish WD, Astephen Wilson JL. Obesity is associated with higher absolute tibiofemoral contact and muscle forces during gait with and without knee osteoarthritis. Clin Biomech (Bristol, Avon). 2016;31:79-86. https://doi.org/10.1016/j.clinbiomech.2015.09.017.
35. DeVita P, Rider P, Hortobagyi T. Reductions in knee joint forces with weight loss are attenuated by gait adaptations in class III obesity. Gait Posture. 2016;45:25-30. https://doi.org/10.1016/j.gaitpost.2015.12.040.

36. Liukkonen MK, Mononen ME, Vartiainen P, Kaukinen $\mathrm{P}$, Bragge $T$, Suomalainen JS, et al. Evaluation of the effect of bariatric surgery-induced weight loss on knee gait and cartilage degeneration. J Biomech Eng. 2018; 140(4). https://doi.org/10.1115/1.4038330.

37. Anandacoomarasamy A, Leibman S, Smith G, Caterson I, Giuffre B, Fransen $M$, et al. Weight loss in obese people has structure-modifying effects on medial but not on lateral knee articular cartilage. Ann Rheum Dis. 2012;71: 26-32. https://doi.org/10.1136/ard.2010.144725.

38. Setton LA, Elliott DM, Mow VC. Altered mechanics of cartilage with osteoarthritis: human osteoarthritis and an experimental model of joint degeneration. Osteoarthritis Cartilage. 1999;7:2-14. https://doi.org/10.1053/ joca.1998.0170.

39. Griffin TM, Fermor B, Huebner JL, Kraus VB, Rodriguiz RM, Wetsel WC, et al. Diet-induced obesity differentially regulates behavioral, biomechanical, and molecular risk factors for osteoarthritis in mice. Arthritis Res Ther. 2010;12: R130. https://doi.org/10.1186/ar3068.

40. Sanchez-Adams J, Leddy HA, McNulty AL, O'Conor CJ, Guilak F. The mechanobiology of articular cartilage bearing the burden of osteoarthritis. Curr Rheumatol Rep. 2014;16:451. https://doi.org/10.1007/s11926-014-0451-6.

41. Coleman MC, Ramakrishnan PS, Brouillette MJ, Martin JA. Injurious loading of articular cartilage compromises chondrocyte respiratory function. Arthritis Rheumatol. 2016;68:662-71. https://doi.org/10.1002/art.39460.

42. Lee W, Leddy HA, Chen Y, Lee SH, Zelenski NA, McNulty AL, et al. Synergy between Piezo 1 and Piezo2 channels confers high-strain mechanosensitivity to articular cartilage. Proc Natl Acad Sci U S A. 2014;111:E5114-22. https:// doi.org/10.1073/pnas.1414298111.

43. Mohanraj B, Meloni GR, Mauck RL, Dodge GR. A high-throughput model of post-traumatic osteoarthritis using engineered cartilage tissue analogs. Osteoarthritis Cartilage. 2014;22:1282-90. https://doi.org/10.1016/j.joca.2014. 06.032 .

44. Honda K, Ohno S, Tanimoto K, ljuin C, Tanaka N, Doi T, et al. The effects of high magnitude cyclic tensile load on cartilage matrix metabolism in cultured chondrocytes. Eur J Cell Biol. 2000;79:601-9. https://doi.org/10. 1078/0171-9335-00089.

45. Guilak F. Biomechanical factors in osteoarthritis. Best Pract Res Clin Rheumatol. 2011;25:815-23. https://doi.org/10.1016/j.berh.2011.11.013.

46. Carman WJ, Sowers M, Hawthorne VM, Weissfeld LA. Obesity as a risk factor for osteoarthritis of the hand and wrist: a prospective study. Am J Epidemiol. 1994; 139:119-29. https://www.ncbi.nlm.nih.gov/pubmed/8296779.

47. Aygun AD, Gungor S, Ustundag B, Gurgoze MK, Sen Y. Proinflammatory cytokines and leptin are increased in serum of prepubertal obese children. Mediat Inflamm. 2005;2005:180-3. https://doi.org/10.1155/MI.2005.180.

48. Wellen KE, Hotamisligil GS. Inflammation, stress, and diabetes. J Clin Invest 2005;115:1111-9. https://doi.org/10.1172/JCI25102.

49. Messier SP, Mihalko SL, Legault C, Miller GD, Nicklas BJ, DeVita P, et al. Effects of intensive diet and exercise on knee joint loads, inflammation, and clinical outcomes among overweight and obese adults with knee osteoarthritis: the IDEA randomized clinical trial. JAMA. 2013;310:1263-73. https://doi.org/10.1001/jama.2013.277669.

50. Huebner JL, Landerman LR, Somers TJ, Keefe FJ, Guilak F, Blumenthal JA, et al. Exploratory secondary analyses of a cognitive-behavioral intervention for knee osteoarthritis demonstrate reduction in biomarkers of adipocyte inflammation. Osteoarthritis Cartilage. 2016;24:1528-34. https://doi.org/10. 1016/j.joca.2016.04.002.

51. McNulty AL, Miller MR, O'Connor SK, Guilak F. The effects of adipokines on cartilage and meniscus catabolism. Connect Tissue Res. 2011;52:523-33. https://doi.org/10.3109/03008207.2011.597902.

52. Hui W, Litherland GJ, Elias MS, Kitson Gl, Cawston TE, Rowan AD, et al. Leptin produced by joint white adipose tissue induces cartilage degradation via upregulation and activation of matrix metalloproteinases. Ann Rheum Dis. 2012;71:455-62. https://doi.org/10.1136/annrheumdis-2011-200372.

53. Eckstein F, Tieschky M, Faber S, Englmeier KH, Reiser M. Functional analysis of articular cartilage deformation, recovery, and fluid flow following dynamic exercise in vivo. Anat Embryol. 1999;200:419-24. http://www.ncbi. nlm.nih.gov/pubmed/10460479. 